\title{
XEN gel implant with or without phacoemulsification for glaucoma: a systematic review and meta-analysis
}

\author{
Bo Wang ${ }^{1,2,3}$, Xiangjie Leng ${ }^{1,2,3}$, Xuemei An ${ }^{4}$, Xiwen Zhang ${ }^{1,2,3}$, Xiang Liu ${ }^{1,2,3}$, Xuejing Lu ${ }^{1,2,3}$ \\ ${ }^{1}$ Department of Ophthalmology, Eye College of Chengdu University of TCM, Chengdu, China; ${ }^{2}$ Department of Ophthalmology, Ineye Hospital of \\ Chengdu University of TCM, Chengdu, China; ${ }^{3}$ Department of Ophthalmology, Key Laboratory of Sichuan Province Ophthalmopathy Prevention \\ \& Cure and Visual Function Protection with TCM, Chengdu, China; ${ }^{4}$ Affiliated Hospital of Chengdu University of Traditional Chinese Medicine, \\ Chengdu, China \\ Contributions: (I) Conception and design: B Wang, X Leng, X An; (II) Administrative support: X Lu; (III) Provision of study materials or patients: B \\ Wang, X Lu; (IV) Collection and assembly of data: B Wang, X Leng, X An, X Liu; (V) Data analysis and interpretation: B Wang, X Leng, X An, X \\ Liu; (VI) Manuscript writing: All authors; (VII) Final approval of manuscript: All authors. \\ Correspondence to: Xuejing Lu, MD. Eye College of Chengdu University of TCM, Chengdu, China. Email: luxuejing@cdutcm.edu.cn.
}

Background: XEN gel implant is an alternative method of treating glaucoma by connecting the anterior chamber and the subconjunctival space. The efficacy of the XEN gel implant and whether to combine with phacoemulsification is what most concerned. This review aims to test the efficacy and safety of the XEN gel implant compared with trabeculectomy and to test the efficacy between XEN combined with phacoemulsification and XEN alone.

Methods: The PubMed, EMBASE, the Cochrane Library, and ClinicalTrials.gov were searched through July 8, 2020. Studies comparing XEN and trabeculectomy or comparing XEN + phacoemulsification and XEN alone were included. The standard mean differences (SMD) were calculated to analyze the lowered intraocular pressure (IOP) and the number of anti-glaucoma medications. All data were measured from baseline to endpoint. The I2 statistic quantified heterogeneity ranged from $0 \%$ to $100 \%$, and a random effects model was used in this meta-analysis. Review Manager 5.3 performed all analyses. The $t$-test calculated all $\mathrm{P}$ values, and $\mathrm{P}$ values were regarded as statistically significant at $\mathrm{P}<0.05$. The methodological index for non-randomized studies was used to find and test bias in the literature.

Results: Twelve studies with 1,602 eyes were included. Five studies compared the XEN gel implant with trabeculectomy. Eight compared XEN + phacoemulsification with XEN alone. There was no significant correlation between XEN and trabeculectomy groups in lowering IOP (SMD 0.30, 95\% CI, 0.00 to 0.60 , $\mathrm{I}^{2}=60 \%$ ) and reduced the number of IOP lowering drugs (SMD 0.01, 95\% CI, -0.16 to $0.17, \mathrm{I}^{2}=0 \%$ ). There was a significant difference between XEN + phacoemulsification and XEN alone in lowering IOP $(1,034$ eyes, SMD $0.22,95 \% \mathrm{CI}, 0.05$ to $0.40, \mathrm{I}^{2}=38 \%$ ) and reducing the number of medications (729 eyes, SMD $0.20,95 \%$ CI, -0.06 to $\left.0.46, \mathrm{I}^{2}=62 \%\right)$.

Conclusions: XEN gel implant, although not effective as trabeculectomy, is a safe operation for openangle and some other types of glaucoma. Meanwhile, XEN alone is more effective than XEN combined with phacoemulsification within 1 week after operations. After three months, the two groups are similar in lowering IOP. More extensive, better-designed, strictly blinded, multicenter randomized clinical trials are needed to confirm our findings.

Keywords: XEN gel implant; trabeculectomy; phacoemulsification; glaucoma

Submitted Aug 10, 2020. Accepted for publication Oct 14, 2020.

doi: 10.21037/atm-20-6354

View this article at: http://dx.doi.org/10.21037/atm-20-6354 


\section{Introduction}

Glaucoma is a disease characterized by progressive loss of retinal nerve fibers and visual field defects and is the leading cause of irreversible visual impairment (1). It is also the second most common cause of blindness in the world $(2,3)$. The number of glaucoma cases worldwide is expected to reach 11.82 million in 2040, with about $60 \%$ of glaucoma cases occurring in Asia (4). The exact pathogenesis of glaucoma is still unclear. Increased intraocular pressure (IOP) as the major factor which may cause damage to retinal ganglion cells. Control IOP by drugs can appropriately delay the progression of glaucoma. For the target IOP, it should be achieved with the least drugs and side effects $(5,6)$. However, the major problems in treatment are patient compliance and ocular surface toxicity (7). When topical medication cannot sufficiently reduce IOP, surgical approaches may be the most effective possibility (8).

Trabeculectomy is the most common incision surgery to reduce IOP (9). It developed to become the goldstandard surgical treatment for glaucoma, as accumulating evidence from trials, including the Collaborative Initial Glaucoma Treatment Study (10) and the Advanced Glaucoma Intervention Study (11). Trabeculectomy is widely performed; however, with limitations, it may cause a series of complications. Complications, including transient intraocular pressure increase, choroidal exudation, iritis, shallow anterior chamber, and anterior chamber hemorrhage may occur early after trabeculectomy (12-15). These complications cause the operation to destroy the ocular surface structure significantly, even if it is successful, except for short-term problems, including nearly $50 \%$ of short-term perioperative complications $(16,17)$, so it is not an ideal treatment.

Drainage of aqueous humor through the formation of filtering bubbles under the conjunctiva is the most widely used surgical procedure in the treatment of glaucoma. Compared with traditional trabeculectomy, glaucoma has received considerable attention in subconjunctival filtration surgery and its drainage device in recent years, and its effectiveness and safety have been well proven $(18,19)$. Minimally invasive glaucoma surgery reduces complications, especially when combined with cataract surgery, its beneficial effect of reducing IOP can reduce or eliminate glaucoma drugs (20). Glaucoma subconjunctival filtration minimally invasive surgery, including Ex-Press micro drainage, nail implantation, drainage valve implantation $(21,22)$, XEN gel implantation, and many others.
The XEN drainage device (Allergan Inc., CA, USA) is a permanent soft mini-drainage tube made of gel material. The XEN GEL Implant is a $6-\mathrm{mm}$ tube with an external diameter of 150 micrometres and an internal diameter of 45 micrometers of collagen-derived gelatin cross-linked with glutaraldehyde, making it permanent and non-degrading, with no foreign body reaction (9). The implant is hard when dry but is designed to be soft and flexible when hydrated, becoming soft within 1 to 2 minutes of implantation (23). By connecting the anterior chamber and the subconjunctival space, it reconstructs the subconjunctival aqueous humor outflow channel, promotes the discharge of anterior aqueous humor, and achieves the effect of reducing IOP $(24,25)$. The XEN gel implants have potentially higher pressure reducing side effects compared to other outflow pathways (9). Recent studies have shown that if XEN is placed in the subconjunctival space, it can protect goblet cells and limit ocular surface inflammation. Compared with trabeculectomy, successful filtration vesicles after XEN gel implantation appear flatter, thinner, and have fewer reflective walls $(26,27)$. The treatment method has slight damage but with high safety and effectiveness.

To date, the XEN gel implant is attracting the attention of many ophthalmologists. The efficacy of the XEN gel implant and whether to combine with phacoemulsification is what most concerned. To the best of our knowledge, there is no meta-analysis on the XEN gel implant. Therefore, this study aims to test the efficacy and safety of the XEN gel implant compared with classic surgery (trabeculectomy) and to test the efficacy between XEN combined with phacoemulsification and XEN alone. We present the following article in accordance with the PRISMA reporting checklist (available at http://dx.doi. org/10.21037/atm-20-6354).

\section{Methods}

\section{Study selection}

The study inclusion criteria were: (I) controlled trials including randomized controlled trials (RCTs), retrospective studies, prospective studies, comparative case studies; (II) studies with at least ten eyes; (III) patients with a confirmed diagnosis of glaucoma; (IV) experimental groups treated by XEN; and (V) studies including the following assessments: IOP and number of medications, which can then be converted into mean \pm SD. The exclusion criteria included: (I) uncontrolled trials, including single-arm 
trials, case reports; (II) studies with less than ten eyes; (III) studies including subjects suffering from non-glaucoma; (IV) studies without XEN; (V) no IOP or the number of medications; (VI) studies without original data (e.g., reviews, comments or letters); (VII) studies not conducted in humans; (VIII) repetitive publications or duplicate data. The same inclusion and exclusion criteria were used in the meta-analysis.

After the first screening, Endnote X9 removed duplicate studies. The remaining studies went through added screening stages, including title screening, abstract screening, and full-text screening. During title and abstract screening, uncorrelated studies were excluded from the analysis. After the full-text screening, studies that did not meet the inclusion/exclusion criteria were excluded from the analysis. Two researchers independently reviewed these studies (Wang B, Leng X), and the third researcher resolved these differences (An X).

\section{Data sources and search strategy}

This systematic review was performed using the Preferred Reporting Items for Systematic Reviews and Meta-Analysis (PRISMA) (28) guidelines. This article is registered in PROSPERO (ID: CRD42020197638. Two researchers (Wang B, Leng X) found 713 articles published before July 8, 2020, through database searching with MeSH/Emtree and entry terms. A total of 713 studies were found in English databases: 98 from PubMed; 586 from EMBASE; 5 from the Cochrane Library. We also searched for 24 relevant studies in ClinicalTrials.gov (www.clinicaltrials. gov). Besides, we review the references of the retrieved articles to find other related studies, if they meet the inclusion criteria.

\section{Quality assessment and data extraction}

The data were extracted and collected back-to-back by two researchers (Wang B, Leng $\mathrm{X}$ ) to avoid bias in the data abstraction process. Any differences were resolved through discussion by the third researcher (Zhang X). The following information was extracted from each trial: the name of the first author, year of publication, country, the number of included eyes, study design, types of glaucoma, age of participants, sex ratio, the baseline of IOP, and the number of medications, and follow-up period. An electronic summary database was set up in Microsoft Word 2016, and the data at each stage were analyzed by Review Manager

\section{3 (RevMan 5.3).}

The methodological index for non-randomized studies (MINORS) was used to test each article, resulting in a necessary evaluation of the overall quality of the literature to assess the quality of studies in this meta-analysis. The total score of MINORS is 24 points. After two researchers (Wang B, Leng $\mathrm{X}$ ) tested the articles independently, all data were compared and discussed, and disagreements were resolved by a third researcher (Liu X).

\section{Data synthesis and analysis}

All analyses were conducted with RevMan 5.3 software. After the data were extracted, a meta-analysis was conducted. During the analysis, standard mean differences (SMD) were calculated to analyze the continuous parameters of IOP and the number of anti-glaucoma medications. All data were measured from baseline to endpoint. Heterogeneity was quantified by the $\mathrm{I}^{2}$ statistics, which was independent of the number of studies included in the cumulative analysis. $\mathrm{I}^{2}$ values range from 0 to $100 \%$, and higher values show a more significant heterogeneity. When heterogeneity was found, a random effects model was used to account for the estimation of interstudy variance and to increase the confidence interval (CI).

\section{Additional analysis}

We performed a sensitivity analysis by excluding each study to observe if the $\mathrm{I}^{2}$ value decreased. Here, the source of heterogeneity was found, and a subgroup analysis was performed. If there was no evident decrease in the $\mathrm{I}^{2}$ value, we performed subgroup analyses of any possible confounding factors, including the follow-up period and baseline of the IOP and the number of medications, to minimize the heterogeneity. We are also summarized as adverse events/complications in table formats.

\section{Results}

We found 713 references from three electronic databases and a supplementary search from ClinicalTrails.gov. After 78 duplicate studies were removed, 635 articles remained and were subjected to added screening. A total of 564 articles were excluded through title and abstract screening. Then, we screened 71 full-text articles for this review, and $31,17,4,4,2$, and 1 studies were excluded because they had no control group, they were reviewed, were not original articles, were not about XEN, were 


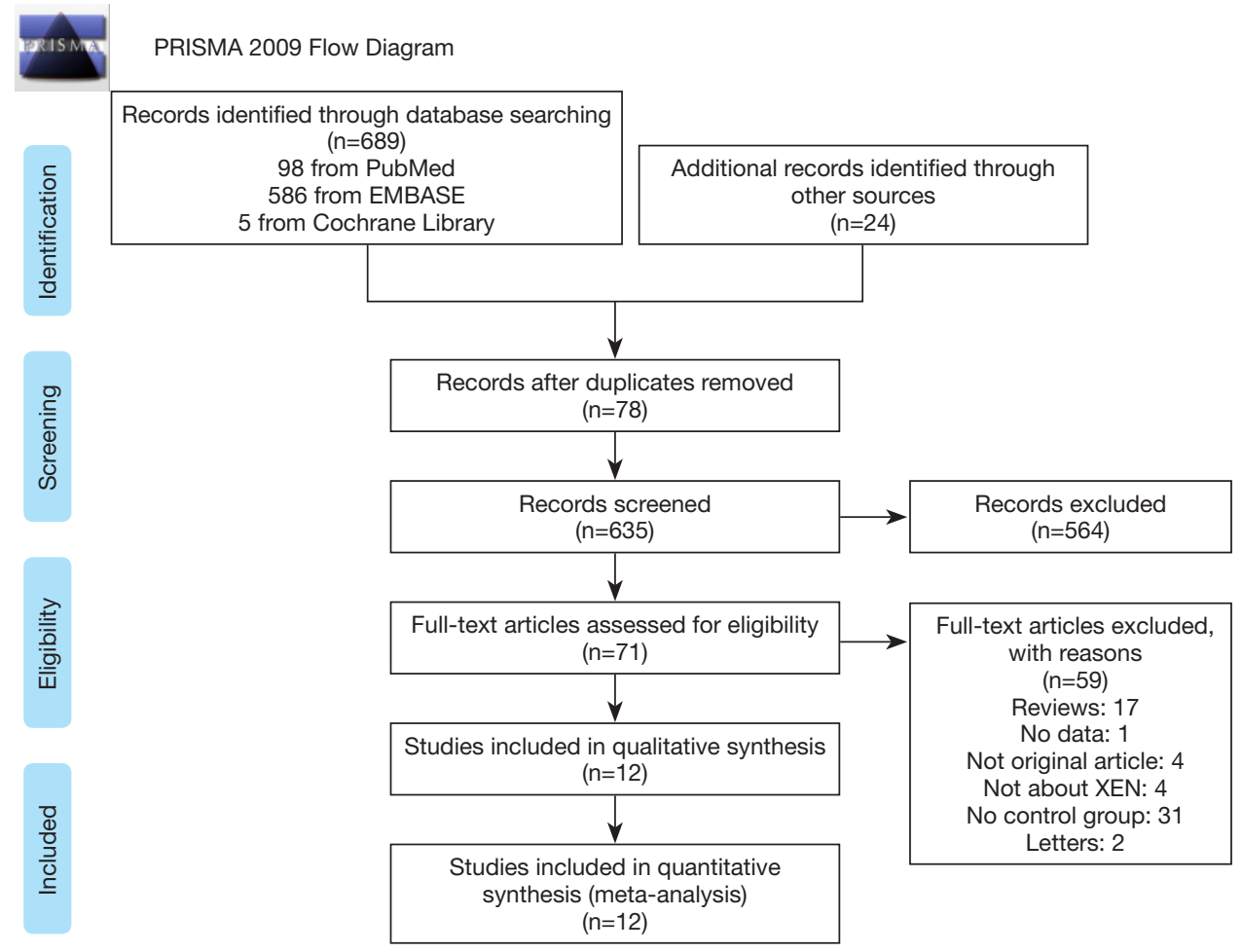

Figure 1 Flow diagram.

letters, and they had no available data, respectively. Finally, we found 12 studies by full-text screening that met the requirements for comprehensive qualitative and quantitative analysis, as shown in Figure 1.

\section{Characteristics and quality of the included studies}

One thousand six hundred two eyes of glaucoma patients distributed from Austria, Canada, Italy, Poland, Spain, Switzerland, Turkey, UK, and the USA were included in the nine included studies. The average age in each RCT ranged from 61.1-78.15, and the most extended follow-up period was two years. All necessary characteristics are summarized in Table 1 (29-40). According to the results of MINORS, the selected studies had a low risk of bias, and the quality of the included studies was high, meeting the current analysis. Specific quality assessment results are also shown in Table 1.

\section{Primary outcomes}

Regarding outcome measures, all included studies examined IOP. Seven recorded the number of medications. From the data of 632 and 555 eyes, there was no significant correlation between XEN and trabeculectomy groups in lowering IOP (SMD 0.30, 95\% CI, 0.00 to $0.60, \mathrm{I}^{2}=60 \%$ ) (Figure 2) and reduce the number of IOP lowering drugs (SMD 0.01, 95\% CI, -0.16 to $0.17, \mathrm{I}^{2}=0 \%$ ) (Figure 3).

\section{Secondary outcomes}

However, between XEN with and without cataract surgery, eight studies examined IOP, and six examined the numbers of medications used by glaucoma patients. There was a significant difference between the two conditions in lowering IOP $\left(1,034\right.$ eyes, SMD $0.22,95 \%$ CI, 0.05 to $\left.0.40, I^{2}=38 \%\right)$ (Figure 4) and reducing the number of medications (729 eyes, SMD $0.20,95 \%$ CI, -0.06 to $0.46, \mathrm{I}^{2}=62 \%$ ) (Figure 5).

\section{Sensitivity analysis}

Comparing XEN (with or without phacoemulsification) and trabeculectomy, the exclusion of the study by Schlenker in 2017 from the meta-analysis significantly altered the pooled estimates of lowered IOP. In particular, the $\mathrm{I}^{2}$ value decreased from $60 \%$ to $0 \%$.

Comparing XEN combined with phacoemulsification 
Table 1 Characteristics of included studies

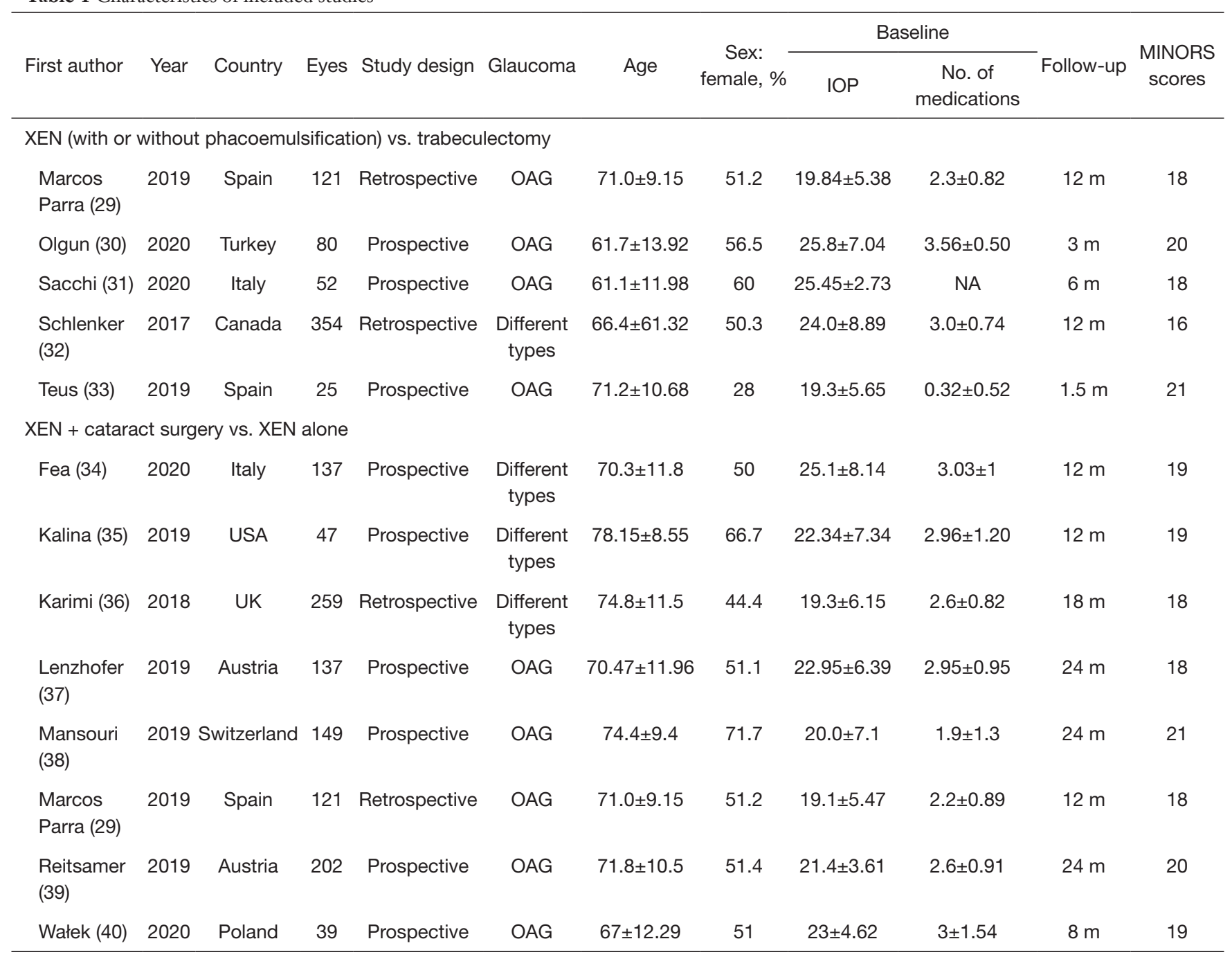

No., number; MINORS, the methodological index for non-randomized studies (total: 24 points); OAG, open-angle glaucoma; m, months.

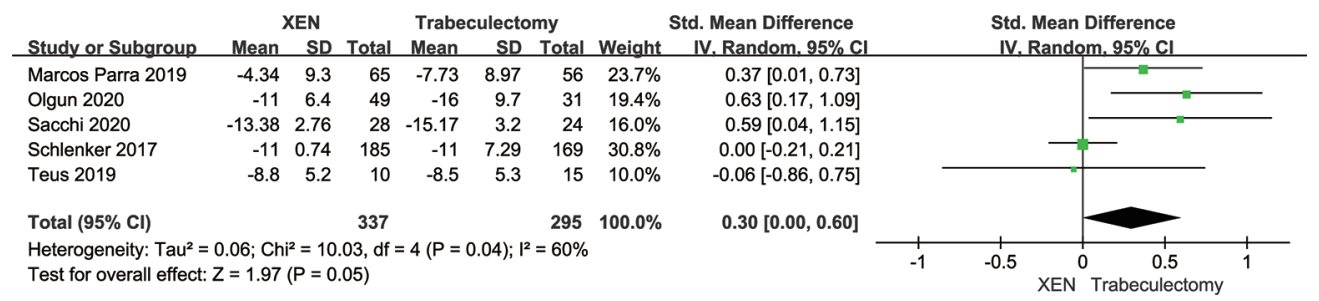

Figure 2 Forest plot of lowering intraocular pressure between XEN gel implant and trabeculectomy.

and XEN alone, the exclusion of the study by Wałek in 2019 from the meta-analysis significantly altered the pooled estimates of the number of anti-glaucoma medications. The $\mathrm{I}^{2}$ value decreased from $62 \%$ to $37 \%$.

\section{Subgroup analysis}

In the subgroup analysis, to minimize the heterogeneity, we analyzed a total of 2-year results to observe the variation 


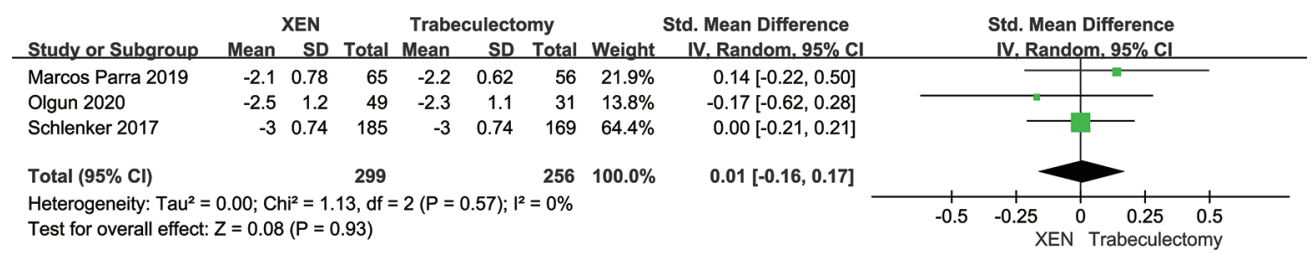

Figure 3 Forest plot of number of anti-glaucoma medications between XEN and trabeculectomy.

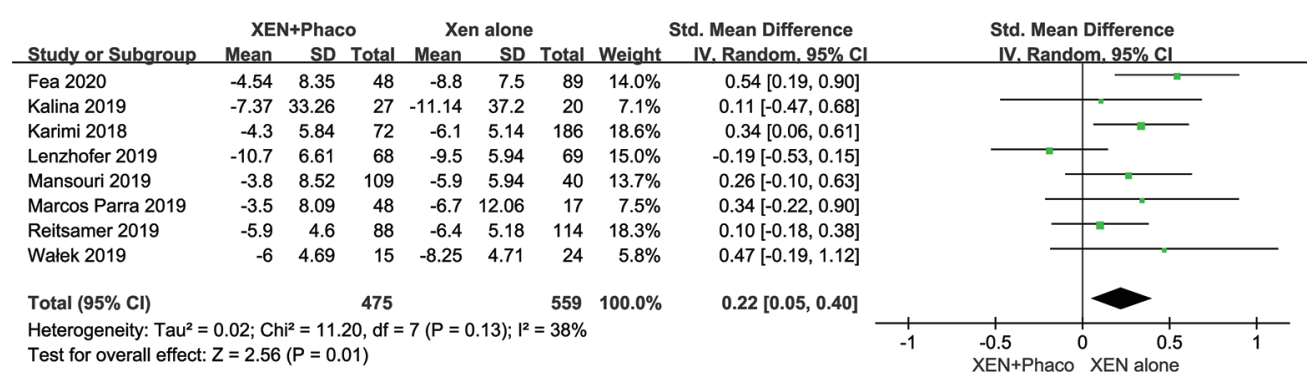

Figure 4 Forest plot of lowering intraocular pressure between XEN gel implant + phacoemulsification and XEN gel implant alone.

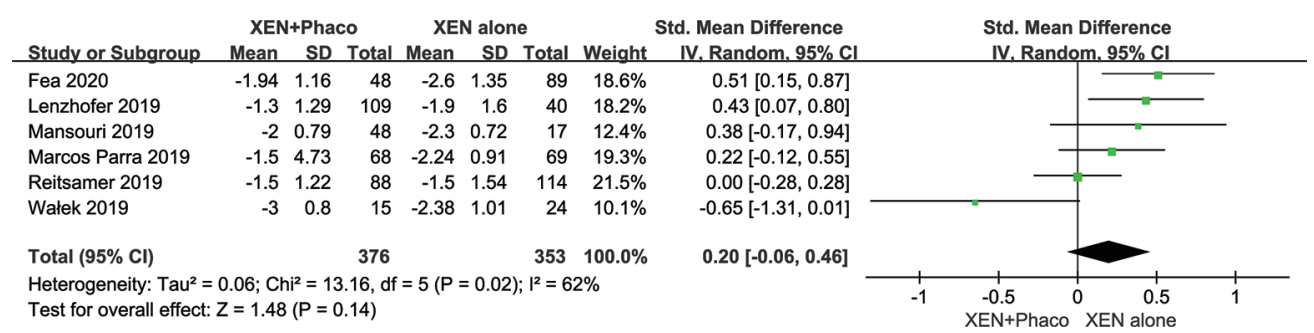

Figure 5 Forest plot of number of anti-glaucoma medications between XEN gel implant + phacoemulsification and XEN gel implant alone.

of IOP in the XEN + phacoemulsification group and XEN group (Figure 6). The single most striking result to emerge from the data comparison was the XEN alone group, which showed superior at the first week (470 eyes, SMD 0.59, 95\% CI, 0.14 to $1.04, I^{2}=62 \%$ ) and six months ( 470 eyes, SMD $0.39,95 \%$ CI, 0.17 to $0.62, \mathrm{I}^{2}=0 \%$ ) after XEN operation. On the contrary, no statistically significant difference in 3 months (470 eyes, SMD $0.13,95 \%$ CI, -0.17 to 0.43 , $\left.\mathrm{I}^{2}=30 \%\right), 1$ year (858 eyes, SMD $0.09,95 \% \mathrm{CI},-0.09$ to $0.27, \mathrm{I}^{2}=30 \%$ ), and 2 years ( 351 eyes, SMD $0.16,95 \% \mathrm{CI}$, -0.06 to $\left.0.38, \mathrm{I}^{2}=0 \%\right)$.

\section{Discussion}

\section{Findings and interpretations}

This meta-analysis of 12 studies, including 1,602 eyes, aimed to investigate the efficacy and safety of XEN gel implant with or without phacoemulsification to treat glaucoma.

The pooled results of the meta-analysis showed there is no statistical difference between XEN gel implant and trabeculectomy on lowering IOP, with high heterogeneity. However, after sensitive analysis excluded the study by Schlenker in 2017 , the high heterogeneity decreased to $0 \%$, for it is the only retrospective study. Meanwhile, the results changed to be statistically different. It turns out that trabeculectomy is more effective in lowering the IOP of glaucoma patients. Meanwhile, there is no significant difference in reducing the number of anti-glaucoma medications between the two groups.

Although the efficacy of the XEN gel implant is not better at lowering IOP compared to trabeculectomy, the efficacy is also acceptable. XEN gel implant has the same efficacy in reducing the number of anti-glaucoma medications. Results from international studies showed 


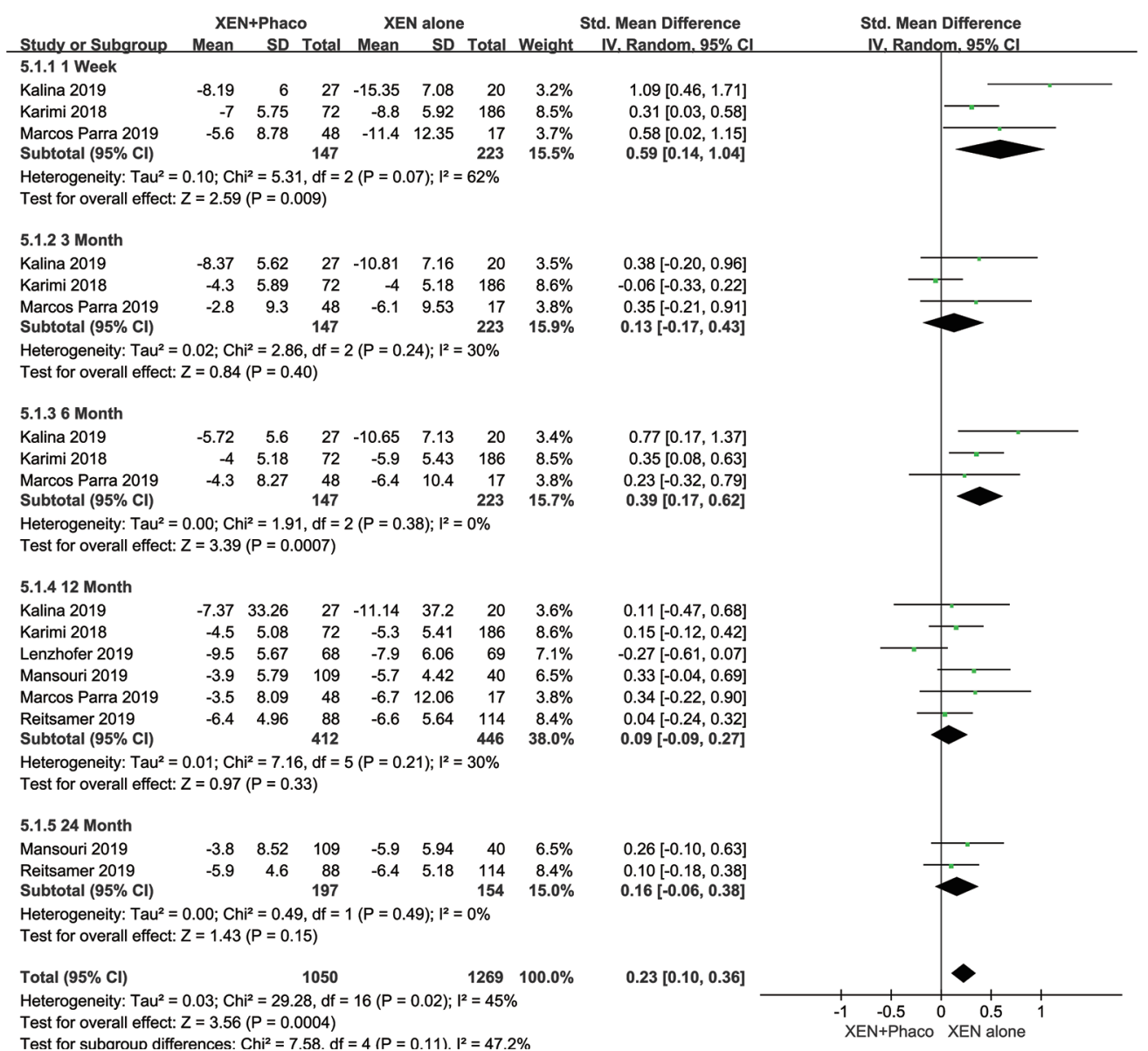

Figure 6 Forest plot of lowering intraocular pressure between XEN gel implant + phacoemulsification and XEN gel implant alone in 1 week, 3, 6, 12, 24 months.

Table 2 The differences between XEN gel implant and trabeculectomy

\begin{tabular}{lcc}
\hline Variable & XEN & Trabeculectomy \\
\hline Lowering IOP & Less & Better \\
Reducing No. of medications & Same & Same \\
Conjunctival injury & Almost none & Yes \\
Scleral flap & No & Yes \\
Anterior chamber & Stabilization & Instability \\
Peripheral iridectomy & No & Yes \\
Wound size & Smaller & Larger \\
Operation complexity & Simpler & More complex \\
Postoperative inflammation & Less & More \\
Scarring & Almost none & More \\
\hline
\end{tabular}

a reduction of $38 \%$ of the mean IOP at 24 months and a reduction of $48 \%$ of medications at 24 months from the greatest medicated IOP (41). As an implant, XEN can reduce surgical risks to the patient and minimize damage from the surgery. The mechanism of the XEN gel implant is like trabeculectomy, and that is to fix the aqueous to flow from the anterior chamber to the subconjunctival space, forming a low-lying and diffuse bleb, considered the major reason leads to no significant difference. One thing should be cautious; the mean baseline of IOP in all included studies are only $26 \mathrm{mmHg}$. We are not sure if it is suitable for glaucoma patients with higher IOP, although the refractory glaucoma is one indication. The differences between XEN gel implant and trabeculectomy are summarized in Table 2. Comparing to trabeculectomy, XEN has obvious advantages in many aspects. XEN has almost no conjunctival injury and scarring, does not need to make scleral flap, and 
Table 3 Adverse event complications of the XEN gel implant

\begin{tabular}{|c|c|c|c|c|c|c|c|c|c|c|c|}
\hline $\begin{array}{l}\text { Adverse } \\
\text { events/ } \\
\text { complications }\end{array}$ & Hyphema & Hypotony & $\begin{array}{l}\text { Hypotonous } \\
\text { maculopathy }\end{array}$ & $\begin{array}{c}\text { Exposed } \\
\text { XEN }\end{array}$ & $\begin{array}{l}\text { Blocked } \\
\text { XEN }\end{array}$ & $\begin{array}{l}\text { Vision } \\
\text { loss }\end{array}$ & $\begin{array}{c}\text { Subconjunctival } \\
\text { hemorrhage }\end{array}$ & $\begin{array}{c}\text { Iris } \\
\text { damage }\end{array}$ & $\begin{array}{c}\text { Choroidal } \\
\text { detachment }\end{array}$ & $\begin{array}{l}\text { Choroidal } \\
\text { effusion }\end{array}$ & $\begin{array}{l}\text { Retinal } \\
\text { disorder }\end{array}$ \\
\hline
\end{tabular}

perform peripheral iridectomy in the operation, while trabeculectomy has the opposite. XEN is better than trabeculectomy in the stability of anterior chamber, the wound size, the operation complexity, and postoperative inflammation. The advantages of XEN are that the anterior chamber of XEN is stable, while trabeculectomy is instable, the wound size of XEN is smaller, and trabeculectomy is larger. XEN has a simpler procedure, trabeculectomy is more complicated, XEN has less postoperative inflammation, and trabeculectomy has more postoperative inflammation. In terms of the amount of postoperative medication, trabeculectomy was equal to $\mathrm{XEN}$, and as for lowering IOP, XEN is not as good as trabeculectomy. With the acceptable decrease of IOP and less adverse events or complications, it is an excellent possibility for OAG or other types of glaucoma (e.g., pseudoexfoliation, glaucoma, pigment dispersion, glaucoma).

Since the XEN gel implant used clinically, whether XEN combined with cataract surgery is more effective than XEN alone, treating glaucoma patients has brought an increasing interest in glaucoma researchers. A good deal of relevant research was conducted in the past three years, as we showed in the results part. XEN alone was more effective in lowering IOP than the combined groups, and lowering the number of anti-glaucoma after excluding the study by Wałek in 2019 through sensitive analysis (40). According to the introduction, the XEN gel implant was designed for the management of refractory glaucoma, including cases where previous surgical treatment has failed, cases of primary open-angle glaucoma, and pseudoexfoliative or pigmentary glaucoma with open angles unresponsive to the maximum tolerated medical therapy. In eight studies, five of them included OAG patients, while the other three studies included several types of glaucoma, in which most patients were OAG patients too. POAG is the most common type of OAG. Lowering the pretreatment IOP by $25 \%$ or more has been shown to delay the progression of POAG (42).

To specifically investigate the factors leading to unexpected results, we performed a subgroup analysis considering the influence of the follow-up period. Within one week, the IOP decrease was significantly different between XEN alone, which lowered more IOP, and XEN combined with phacoemulsification. Fea et al. also noted that the differences were clinically meaningful (34). However, to our knowledge, the unexpected results are still unexplained. We assumed that this was attributed to phacoemulsification surgery. In combined groups, viscoelastics were used in the surgery and were removed at the end of the XEN gel implant operation (43). The residues of viscoelastics may cause the XEN gel implanted to unsmooth, leading to the difference between the two groups. After three months, the residues may be absorbed or flow through the XEN gel implant, and therefore, there is no significant difference between the two groups. Meanwhile, more factors, including the age of patients (the elders have more age-related cataract patients), the baseline of IOP and the number of anti-glaucoma medications, and types of glaucoma should be considered.

We extracted eight included studies, reported adverse events/complications, and summarized numbers of occurrences across the articles in Table 3. The adverse events or complications must be reported at least twice. Hyphema, mentioned by five articles, was the most common complication. Hypotony, hypotonous maculopathy, and exposed XEN were reported 4, 3, and 3 times, respectively. As for blocking XEN, vison loss, subconjunctival hemorrhage, iris damage, choroidal detachment, choroidal effusion, and retinal disorder were only mentioned twice. For glaucoma experts, postoperative scarring of a surgery is surely an important problem. XEN is a minimally invasive glaucoma surgery (MIGS). Generally, only few articles report postoperative blebs and scarring. Because most of the included studies use 5-fluorouracil (5-FU) and Mitomycin $\mathrm{C}(\mathrm{MMC})$ to lessen postoperative scarring.

In the future, for open-angled patients, MIGS may become the main method treating glaucoma due to low risk, simplized operation and minor damage of eye ball. Expectably, it will not be the end of MIGS, because MIGS 
may develop to be used in angle-closure glaucoma and malignant glaucoma patients.

\section{Limitations}

Some limitations should be considered in this metaanalysis. The analyzed studies have significant differences in patient characteristics, including age, the baseline of IOP, and the number of anti-glaucoma medications, and the difference in types of glaucoma, all of which may affect the results. All included studies were non-RCTs, although with high quality tested by MINORS, further studies should concentrate more on randomized prospective trails, otherwise unique characteristics would make it more challenging to investigate the heterogeneity. Meanwhile, the mean baseline IOP of included studies were only $26 \mathrm{mmHg}$. It was unlikely to show the efficacy in patients with higher IOP. Because of insufficient data of adverse events/complications in the comparison between XEN gel implant and trabeculectomy, the only primary description was available in the present study.

\section{Conclusions}

XEN gel implant, although not effective as trabeculectomy, is a safe operation for OAG and some other types of glaucoma. Meanwhile, XEN alone is more effective than XEN combined with phacoemulsification within one week after operations. After three months, the two groups are similar in lowering IOP, and the IOP becomes stable. Because of the limitations of the included studies, larger, well-designed, strictly blinded, multicenter RCTs are needed to confirm our findings.

\section{Acknowledgments}

Funding: This study was supported by the Sichuan Youth Innovation Team of Traditional Chinese Medicine Industry (No. 2017TD0030).

\section{Footnote}

Reporting Checklist: The authors have completed the PRISMA reporting checklist. Available at http://dx.doi. org/10.21037/atm-20-6354

Conflicts of Interest: All authors have completed the ICMJE uniform disclosure form (available at http://dx.doi. org/10.21037/atm-20-6354). The authors have no conflicts of interest to declare.

Ethical Statement: The authors are accountable for all aspects of the work in ensuring that questions related to the accuracy or integrity of any part of the work are appropriately investigated and resolved. This article does not have any studies with human participants or animals performed by any of the authors.

Open Access Statement: This is an Open Access article distributed in accordance with the Creative Commons Attribution-NonCommercial-NoDerivs 4.0 International License (CC BY-NC-ND 4.0), which permits the noncommercial replication and distribution of the article with the strict proviso that no changes or edits are made and the original work is properly cited (including links to both the formal publication through the relevant DOI and the license). See: https://creativecommons.org/licenses/by-nc-nd/4.0/.

\section{References}

1. Conlon R, Saheb H, Ahmed II. Glaucoma treatment trends: a review. Can J Ophthalmol 2017;52:114-24.

2. Gupta V, Dada T. Should we perform peripheral laser iridotomy in primary angle closure suspects: implications of the ZAP trial? Ann Transl Med 2019;7:S157.

3. Jonas JB, Aung T, Bourne RR, et al. Glaucoma. Lancet 2017;390:2183-93.

4. Tham YC, Li X, Wong TY, et al. Global prevalence of glaucoma and projections of glaucoma burden through 2040: a systematic review and meta-analysis. Ophthalmology 2014;121:2081-90.

5. Marshall LL, Hayslett RL, Stevens GA. Therapy for Open-Angle Glaucoma. Consult Pharm 2018;33:432-45.

6. Weinreb RN, Aung T, Medeiros FA. The pathophysiology and treatment of glaucoma: a review. JAMA 2014;311:1901-11.

7. Lemij HG, Hoevenaars JG, van der Windt C, et al. Patient satisfaction with glaucoma therapy: reality or myth? Clin Ophthalmol 2015;9:785-93.

8. Kennedy JB, SooHoo JR, Kahook MY, et al. Selective Laser Trabeculoplasty: An Update. Asia Pac J Ophthalmol (Phila) 2016;5:63-9.

9. Chaudhary A, Salinas L, Guidotti J, et al. XEN Gel Implant: a new surgical approach in glaucoma. Expert Rev Med Devices 2018;15:47-59.

10. Musch DC, Lichter PR, Guire KE, et al. The 
Collaborative Initial Glaucoma Treatment Study: study design, methods, and baseline characteristics of enrolled patients. Ophthalmology 1999;106:653-62.

11. Investigators A. The Advanced Glaucoma Intervention Study (AGIS): 7. The relationship between control of intraocular pressure and visual field deterioration. The AGIS Investigators. Am J Ophthalmol 2000;130:429-40.

12. Gedde SJ, Herndon LW, Brandt JD, et al. Postoperative complications in the Tube Versus Trabeculectomy (TVT) study during five years of follow-up. Am J Ophthalmol 2012;153:804-14.e1.

13. Gedde SJ, Singh K, Schiffman JC, et al. The Tube Versus Trabeculectomy Study: interpretation of results and application to clinical practice. Curr Opin Ophthalmol 2012;23:118-26.

14. Song J. Complications of selective laser trabeculoplasty: a review. Clin Ophthalmol 2016;10:137-43.

15. Guven Yilmaz S, Palamar M, Yusifov E, et al. Effects of primary selective laser trabeculoplasty on anterior segment parameters. Int J Ophthalmol 2015;8:954-9.

16. Jampel HD, Musch DC, Gillespie BW, et al. Perioperative complications of trabeculectomy in the collaborative initial glaucoma treatment study (CIGTS). Am J Ophthalmol 2005;140:16-22.

17. Gedde SJ, Schiffman JC, Feuer WJ, et al. Treatment outcomes in the Tube Versus Trabeculectomy (TVT) study after five years of follow-up. Am J Ophthalmol 2012;153:789-803.e2.

18. Giovingo M. Complications of glaucoma drainage device surgery: a review. Semin Ophthalmol 2014;29:397-402.

19. Pillunat LE, Erb C, Jünemann AG, et al. Micro-invasive glaucoma surgery (MIGS): a review of surgical procedures using stents. Clin Ophthalmol 2017;11:1583-600.

20. Fingeret M, Dickerson JE Jr. The Role of Minimally Invasive Glaucoma Surgery Devices in the Management of Glaucoma. Optom Vis Sci 2018;95:155-62.

21. Netland PA, Sarkisian SR Jr, Moster MR, et al. Randomized, prospective, comparative trial of EX-PRESS glaucoma filtration device versus trabeculectomy (XVT study). Am J Ophthalmol 2014;157:433-40.e3.

22. Good TJ, Kahook MY. Assessment of bleb morphologic features and postoperative outcomes after Ex-PRESS drainage device implantation versus trabeculectomy. Am J Ophthalmol 2011;151:507-13.e1.

23. Lewis RA. Ab interno approach to the subconjunctival space using a collagen glaucoma stent. J Cataract Refract Surg 2014;40:1301-6.

24. Dervenis N, Mikropoulou AM, Dervenis P, et al.
Dislocation of a previously successful XEN glaucoma implant into the anterior chamber: a case report. BMC Ophthalmol 2017;17:148.

25. Dupont G, Collignon N. New surgical approach in primary open-angle glaucoma: xen gel stent a minimally invasive technique. Rev Med Liege 2016;71:90-3.

26. Baiocchi S, Mazzotta C, Sgheri A, et al. In vivo confocal microscopy: qualitative investigation of the conjunctival and corneal surface in open angle glaucomatous patients undergoing the XEN-Gel implant, trabeculectomy or medical therapy. Eye Vis (Lond) 2020;7:15.

27. Karimi A, Hopes M, Martin KR, et al. Efficacy and Safety of the Ab-interno Xen Gel Stent After Failed Trabeculectomy. J Glaucoma 2018;27:864-8.

28. Moher D, Liberati A, Tetzlaff J, et al. Preferred reporting items for systematic reviews and meta-analyses: the PRISMA statement. PLoS Med 2009;6:e1000097.

29. Marcos Parra MT, Salinas López JA, López Grau NS, et al. XEN implant device versus trabeculectomy, either alone or in combination with phacoemulsification, in open-angle glaucoma patients. Graefes Arch Clin Exp Ophthalmol 2019;257:1741-50.

30. Olgun A, Duzgun E, Yildiz AM, et al. XEN Gel Stent versus trabeculectomy: Short-term effects on corneal endothelial cells. Eur J Ophthalmol 2020:1120672120924339.

31. Sacchi M, Agnifili L, Brescia L, et al. Structural imaging of conjunctival filtering blebs in XEN gel implantation and trabeculectomy: a confocal and anterior segment optical coherence tomography study. Graefes Arch Clin Exp Ophthalmol 2020;258:1763-70.

32. Schlenker MB, Gulamhusein H, Conrad-Hengerer I, et al. Efficacy, Safety, and Risk Factors for Failure of Standalone Ab Interno Gelatin Microstent Implantation versus Standalone Trabeculectomy. Ophthalmology 2017;124:1579-88.

33. Teus MA, Paz Moreno-Arrones J, Castaño B, et al. Optical coherence tomography analysis of filtering blebs after long-term, functioning trabeculectomy and XEN@ stent implant. Graefes Arch Clin Exp Ophthalmol 2019;257:1005-11.

34. Fea AM, Bron AM, Economou MA, et al. European study of the efficacy of a cross-linked gel stent for the treatment of glaucoma. J Cataract Refract Surg 2020;46:441-50.

35. Kalina AG, Kalina PH, Brown MM. XEN(®) Gel Stent in Medically Refractory Open-Angle Glaucoma: Results and Observations After One Year of Use in the United States. Ophthalmol Ther 2019;8:435-46. 
36. Karimi A, Lindfield D, Turnbull A, et al. A multicentre interventional case series of 259 ab-interno Xen gel implants for glaucoma, with and without combined cataract surgery. Eye (Lond) 2019;33:469-77.

37. Lenzhofer M, Strohmaier C, Hohensinn M, et al. Change in visual acuity 12 and 24 months after transscleral ab interno glaucoma gel stent implantation with adjunctive Mitomycin C. Graefes Arch Clin Exp Ophthalmol 2019;257:2707-15.

38. Mansouri K, Bravetti GE, Gillmann K, et al. TwoYear Outcomes of XEN Gel Stent Surgery in Patients with Open-Angle Glaucoma. Ophthalmol Glaucoma 2019;2:309-18.

39. Reitsamer H, Sng C, Vera V, et al. Two-year results of a multicenter study of the ab interno gelatin implant in medically uncontrolled primary open-angle glaucoma. Graefes Arch Clin Exp Ophthalmol 2019;257:983-96.

Cite this article as: Wang B, Leng X, An X, Zhang X, Liu X, Lu X. XEN gel implant with or without phacoemulsification for glaucoma: a systematic review and meta-analysis. Ann Transl Med 2020;8(20):1309. doi: 10.21037/atm-20-6354
40. Wałek E, Przeździecka-Dołyk J, Helemejko I, et al. Efficacy of postoperative management with 5-fluorouracil injections after XEN Gel Stent implantation. Int Ophthalmol 2020;40:235-46.

41. Vera VI, Horvath C. XEN Gel Stent: The Solution Designed by AqueSys®. In: Samples JR, Ahmed IIK, editors. Surgical Innovations in Glaucoma. New York, NY: Springer New York; 2014. p. 189-98.

42. Prum BE, Jr., Rosenberg LF, Gedde SJ, et al. Primary Open-Angle Glaucoma Preferred Practice Pattern(®) Guidelines. Ophthalmology 2016;123:P41-p111.

43. Sng CCA, Chew PTK, Htoon HM, et al. Case Series of Combined XEN Implantation and Phacoemulsification in Chinese Eyes: One-Year Outcomes. Adv Ther 2019;36:3519-29.

(English Language Editor: J. Chapnick) 